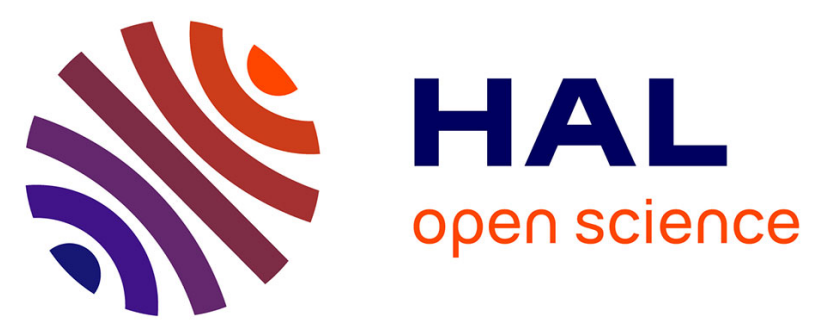

\title{
Optimizing 4-dimensional magnetic resonance imaging data sampling for respiratory motion analysis of pancreatic tumors
}

Bjorn Stemkens, Rob Tijssen, Baudouin Denis de Senneville, Hanne D Heerkens, Marco van Vulpen, Jan Lagendijk, C. van den Berg

\section{To cite this version:}

Bjorn Stemkens, Rob Tijssen, Baudouin Denis de Senneville, Hanne D Heerkens, Marco van Vulpen, et al.. Optimizing 4-dimensional magnetic resonance imaging data sampling for respiratory motion analysis of pancreatic tumors. International Journal of Radiation Oncology, Biology, Physics, 2015, 91 (3), pp.571-578. 10.1016/j.ijrobp.2014.10.050 . hal-01063778

\section{HAL Id: hal-01063778 https://hal.science/hal-01063778}

Submitted on 6 Sep 2017

HAL is a multi-disciplinary open access archive for the deposit and dissemination of scientific research documents, whether they are published or not. The documents may come from teaching and research institutions in France or abroad, or from public or private research centers.
L'archive ouverte pluridisciplinaire HAL, est destinée au dépôt et à la diffusion de documents scientifiques de niveau recherche, publiés ou non, émanant des établissements d'enseignement et de recherche français ou étrangers, des laboratoires publics ou privés. 
Optimizing 4-dimensional magnetic resonance imaging data sampling for respiratory motion analysis of pancreatic tumors

\section{Running Title:}

Optimizing 4D-MRI for motion analysis

Authors:

Bjorn Stemkens, $\mathrm{MSc}^{1}$,

Rob H Tijssen, DPhil ${ }^{1}$,

Baudouin D de Senneville, $\mathrm{PhD}^{2,3}$,

Hanne D Heerkens, MD ${ }^{1}$,

Marco van Vulpen, $\mathrm{MD}, \mathrm{PhD}^{1}$,

Jan JW Lagendijk, PhD ${ }^{1}$,

Cornelis AT van den Berg, $\mathrm{PhD}^{1}$

\section{$\underline{\text { Affiliations }}$}

1) Department of Radiotherapy, University Medical Center Utrecht, Utrecht, The Netherlands

2) Imaging Division, University Medical Center Utrecht, Utrecht, The Netherlands

3) IMB, UMR5251 CNRS/University of Bordeaux, Bordeaux, France

Corresponding Author:

Bjorn Stemkens

Address: Department of Radiotherapy, University Medical Center Utrecht, Q.00.118, Heidelberglaan 100, 3584CX, Utrecht, The Netherlands

Phone: $+31(0) 887553035$

Fax: +31(0)88 7555850

E-mail: b.stemkens@umcutrecht.nl

Acknowledgment of financial support

None

Conflict of interest:

None 


\section{Abstract}

Purpose: To determine the optimum sampling and binning strategy for retrospective reconstruction of 4D-MR data for non-rigid motion characterization of tumor and organs at risk for radiotherapy purposes.

Material and Methods: For optimization, we compared two surrogate signals (external respiratory bellows and internal MR navigator), three binning methods (absolute amplitude, phase and a hybrid method, relative amplitude binning) and two MR sampling strategies (cartesian and radial) in terms of efficiency, image quality and robustness. Using the optimized protocol, three pancreatic cancer patients were scanned to calculate the $4 \mathrm{D}$ motion. ROI analysis was performed to characterize the respiratory induced motion of the tumor and organs at risk simultaneously.

Results: The MRI navigator was found to be a more reliable surrogate for pancreatic motion than the respiratory bellows signal. Relative amplitude binning offered the best binning in terms of filling efficiency and intra-phase amplitude variation. Radial sampling is most benign for undersampling artifacts and intra-view motion. Motion characterization revealed inter-organ and inter-patient variation, as well as heterogeneity within the tumor.

Conclusions: A robust 4D-MRI method, based on clinically available protocols, is presented and successfully applied to characterize the abdominal motion in pancreatic cancer patients.

Key Words: 4D-MRI; radiotherapy; retrospective reconstruction; 4D-CT; non-rigid registration; pancreatic cancer; respiratory motion 


\section{Summary}

We demonstrate a novel MRI method for simultaneous 4D motion analysis of abdominal tumors and organs at risk. Seven healthy subjects and three patients were scanned and motion trajectories were calculated for the tumor and duodenum. The results demonstrated that the proposed technique was able to accurately calculate motion trajectories within a clinically acceptable timeframe. 


\section{Introduction}

Radiotherapy treatments for pancreatic cancer is challenging due to motion, primarily induced by respiration. Conventionally, margins are used to ensure full dose coverage of the target at the cost of extra radiation to nearby organs at risk (OARs). Prior knowledge about patient specific motion, however, may lead to more conformal radiation plans [1].

Currently, 4D-CT is the method of choice for motion characterization in radiotherapy. Unfortunately, CT has limited soft tissue contrast and 4D-CT often suffers from volume inconsistencies (e.g. duplicating, incomplete, or overlapping structures) caused by discrepancies between the breathing frequency and the pitch [2,3]. Magnetic Resonance Imaging (MRI), on the other hand, has superior soft tissue contrast, allowing better differentiation between tumor and surrounding tissue. Moreover, the flexibility of image acquisition in MRI could be used to overcome some of the image artifacts typical of 4D-CT.

Previous authors have reported MRI based motion characterization using 2D-cine MRI [4-7]. However, these methods solely provide tumor motion characteristics, and do not provide information of the OARs. Consequently, retrospective binning methods have gained interest recently. To bin the acquired data into appropriate respiratory phases, a number of respiratory surrogates have been investigated, such as respiratory bellows [4,8], or image based body area [9]. Another method is to use a 1D MRI navigator that records the diaphragm position [10].

To construct 4D-MR data, previous studies have reported retrospective stacking of 2D multi-slice images using different respiratory surrogates $[8,9,11,12]$. These methods are closely related to 4D-CT and therefore suffer from similar volume inconsistencies. Volumetric 3D acquired MRI scans overcome these problems, since the entire volume is excited each time. Moreover, signal-to-noise ratio (SNR) is inherently larger for 3D acquisitions. Buerger et al. 
[13] used a custom $3 \mathrm{D}$ read-out and an extensive reconstruction process $( \pm 7.5 h)$ to create multiple 3D volumes, which is undesirable for clinical practice.

The goal of this study is to develop a 4D-MRI method to characterize respiratory induced motion of pancreatic tumors and its surrounding OARs using clinically available scan protocols. For this purpose, two motion surrogate signals that serve as input signal for the binning process are assessed. Moreover, two MRI sampling strategies and three binning methods are explored in terms of efficiency, imaging time and image quality. The final resulting technique is applied to three pancreatic cancer patients for motion characterization using a 3D optical flow (OF) algorithm, because of its short processing time and minimal number of control parameters [14].

\section{Methods}

We performed three different experiments in which the surrogate signal, MRI sampling strategy and binning method are independently optimized. The optimized protocol was used to scan three pancreatic cancer patients. Subsequently 3D motion of ten respiratory phases is calculated for both the tumor and the duodenum. All experiments are conducted on a $1.5 \mathrm{~T}$ Philips Achieva MR scanner (Philips Healthcare, Best, The Netherlands). Processing is performed using MATLAB (The Mathworks, Natick, MA).

\subsection{Surrogate signal}

Two healthy subjects were scanned using a fast, coronal, 2D transient balanced steady-state gradient echo (balanced Turbo Field Echo, bTFE) sequence with radial read-out $\left(\alpha=30^{\circ}\right.$, TE/TR $=1.3 / 2.6 \mathrm{~ms}, \mathrm{FOV}=294 \times 294 \mathrm{~mm}^{2}$, voxelsize $=1.5 \times 1.5 \mathrm{~mm}^{2}$, turbo-factor $=128$, 500 dynamics, temporal frequency $2.6 \mathrm{~Hz}$ ). The imaging plane was angulated parallel to the spine to sample along the principal axis of pancreatic motion, thereby reducing through-plane 
motion. The image acquisition was interleaved with a 1D MRI navigator, which was positioned at the dome of the right hemi-diaphragm and respiratory bellows, provided by the MRI vendor, was placed on the upper abdomen.

To assess the agreement of the two surrogates with the pancreatic motion, we performed a general linear model (GLM) analysis, which is a least-squares fit to the data described by $Y=X \beta+\epsilon$. Here $Y$ is the pancreatic motion, obtained by a 2D OF algorithm [15-17], $\beta$ the

parameter estimates, $X$ the surrogate signal and $\epsilon$ represents the residual error. Spatial residual error $(\epsilon)$ maps were calculated to find the surrogate with the least residuals. Furthermore, power spectrum analysis (PSA) was performed on the surrogate signals and the pancreatic motion to get insight in the agreement in frequency range of the surrogates with the pancreatic motion.

\subsection{Binning Simulations}

Another important consideration is how to reorder the continuously acquired MR data with respect to the respiratory cycle (i.e. retrospective binning). 4D-CT scanners employ two binning methods. Absolute amplitude and phase binning (see Fig. 1b). In the first method, the ten bins (i.e., respiratory phases) are determined by the minimum and maximum of the entire respiratory signal. The bins are thus fixed throughout the reordering process. The disadvantage of this method is that, when the breathing amplitude varies, certain phases will be sparsely sampled $[2,18]$. The phase binning method determines two consecutive exhale peaks and defines ten phases with equal time spacing. The disadvantage of this method, however, is that for an irregular breathing pattern, the amplitude variation within each bin can be much larger compared to absolute amplitude binning [19]. To address these disadvantages, we introduce a third, hybrid, binning method, relative amplitude binning. This method defines the maximum exhale and inhale for each respiratory cycle and normalizes them before defining ten 
respiratory phases. We further distinguish between in- and exhale to be able to model hysteresis (i.e. varying motion paths for in- and exhale).

As retrospective reordering of continuously acquired data is a stochastic process, the binning method directly influences the dynamics of the reordering and thus the number of (continuously acquired) volumes required to fill each of the bins with sufficient data. To get insight into how quickly the bins are filled for each of the three binning methods, we performed various simulations. The navigator signal, which was acquired in the subsequent 3D in-vivo experiments, was used as input (8:35 minutes). The time to fill a percentage of all $k$-space lines required for the ten reconstructed volumes was calculated for an increasing number of acquired volumes. Moreover, to evaluate the intra-phase amplitude variation (which should be as small as possible) for the three binning methods, the mean amplitude and standard deviation for each phase was calculated based on the navigator amplitude.

\subsection{In-vivo experiments}

Seven healthy volunteers and three patients with an unresectable pancreas tumor provided written informed consent to be scanned according to institutional rules. A clinically available 3D bTFE was used with turbo direction along $k z$ and a shot length equal to the amount of slices, acquiring all $\mathrm{kz}$ lines within a single shot (see Fig. 1a). Prior to each shot, a navigator was acquired.

We compared cartesian and radial in-plane sampling, using the parameters described in Table 1, in terms of image quality and robustness. 25 Dynamics were acquired as a concession between imaging time and filling. One subject was scanned with the cartesian protocol, whereas the other subjects and patients were scanned with the radial protocol. Two subjects, who were scanned with the radial protocol, had a smaller FOV (330x330x64 $\left.\mathrm{mm}^{3}\right)$, which was sufficient to cover the pancreatic motion path. 
Reconstruction was performed offline on an 8-core CPU computer using ReconFrame reconstruction software (Gyrotools, Zurich, $\mathrm{CH}$ ). Due to the stochastic nature of the filling of respiratory phases, it is likely that certain $k$-space segments are acquired multiple times during acquisition. When this occurred, complex averaging was performed to increase SNR. Image quality was assessed both visually and using the structural similarity (SSIM) index [20], which calculates the similarity between two images as a ratio between 0 (no similarity) and 1 (perfect similarity).

\subsection{D Motion Analysis}

Data of three patients were reconstructed using relative amplitude binning. Non-rigid motion was calculated using a multi-threaded optimized 3D OF algorithm [14]. In short, this algorithm calculates the motion between two imaging frames based on intensity gradients with an additional constraint on motion smoothness to model elastic organ deformation. All respiratory phases were registered to one reference phase (the first phase) as can be seen in Fig. 1c. The tumor and OARs were delineated on the reconstructed reference volume, and the mean motion within each delineated structure was calculated for all respiratory phases.

To assess the quality of the motion estimation by the OF algorithm, all images were registered to the reference phase and visually inspected for residual motion. Moreover, the motion vectors of consecutive phases were added. Since this trajectory comprises a full respiratory cycle, the results should be close to zero. Furthermore, the OF results were compared to results obtained by Elastix [21]. Elastix is a widely used ITK-based non-rigid registration package, which uses B-spline registration, but requires much more computation time, and can therefore not be used in an online setting.

\section{Results}




\subsection{Surrogate signal}

Fig. 2 a shows the residuals of the GLM using either the respiratory bellows or the MRI navigator as surrogate signal to model the motion within the abdomen for a representative subject along with an anatomical reference image. It is seen that the residuals within the pancreas are 3 times higher for the bellows compared to when the navigator is used. These maps further demonstrate the large motion heterogeneity over the FOV. Fig. 2b displays the power spectra of the pancreatic motion, and the spectra of the two surrogates. Aside from large respiratory peaks between 0.25 and $0.32 \mathrm{~Hz}$ in all spectra, low frequencies $(<0.05 \mathrm{~Hz})$ are present in the spectra of the pancreatic motion and the navigator, but absent in the bellows spectrum, which may indicate that the bellows are unable to pick up slow frequency position drifts. A diaphragm navigator is therefore used for all subsequent experiments.

\subsection{Binning Simulations}

Fig. 3a illustrates the amplitude variation within each phase for the three binning methods. Absolute amplitude binning displays the smallest intra-phase variation, with a mean standard deviation over all subjects and phases of $2.0 \mathrm{~mm}$. Phase binning reveals the largest amplitude variation, with a mean standard deviation of $3.9 \mathrm{~mm}$, whereas relative amplitude binning has a mean standard deviation of $3.3 \mathrm{~mm}$. Fig. $3 \mathrm{~b}$ shows the ratio of the total $k$-space that is assigned to its appropriate respiratory phase as a function of the acquired number of dynamics. It reveals a rapid increase in $k$-space filling in the beginning, but an asymptotical approach towards $100 \%$ due to the stochastic character of the respiratory filling. The average filling over all subjects after 25 dynamics is $74 \% \pm 4.4 \%$ for absolute amplitude, $92 \% \pm 2.5 \%$ for relative amplitude, and $92 \% \pm 2.4 \%$ for phase binning. Fig. 3c, finally, shows the distribution of the assigned $k$-space data across the ten phases. Missing data are denoted in white. The figure clearly demonstrates that the inhale phases (i.e. phase five/six) are only sparsely sampled with data when absolute amplitude binning is used. Considering the trade-off between low 
intra-phase amplitude variation and uniform $k$-space filing, relative amplitude binning will be used for the subsequent experiments.

\subsection{In-vivo experiments}

Fig. 4a displays the reconstructed images of the first phase (exhale) for cartesian and radial sampling using a varying amount of dynamics. Cartesian sampled images reveal ghosting artifacts for all reconstructions. Radially sampled images show minor streaking artifacts when 10 dynamics are used, which are mitigated when more dynamics are included. SSIM values between 10 and 20 dynamics and the full reference (i.e. 50 dynamics for cartesian, 25 dynamics for radial) are 0.64 and 0.69 for cartesian and 0.76 and 0.95 for radial. This shows that radial sampling is more benign for undersampling, revealing fewer artifacts. Fig. $4 \mathrm{~b}$ displays the reconstructed images for phase five (inhale), which is difficult to reconstruct due to large intra-phase variation. Despite $100 \%$ filling, the cartesian sampled images still experience ghosting artifacts, as a result of intra-view motion. The image quality of the radially sampled image, on the other hand, is comparable to the first phase.

\subsection{D Motion Analysis}

Fig. 5 shows a coronal and sagittal slice with the vectorfield displayed on top in panels a-b along with the cranio-caudal (CC) motion of the inhale phase with respect to the reference phase in panel c for one patient. Panels d-e show motion trajectories of the tumor and duodenum for all patients. The vectorfields demonstrate the motion heterogeneity within the FOV. Fig. 5c reveals large CC motion for the tumor and the OARs, whereas the motion around the spine and closer to the body contour is significantly less. Substantial differences in CC motion are observed for the two kidneys. AP motion is considerably less, with the largest anterior motion at the location of the psoas muscle, affecting both kidneys. LR motion is marginal in all structures. Furthermore, heterogeneity of motion is observed within the tumor 
(arrow). As the tumor shown here was unresectable, the heterogeneity could result from the observed ingrowth into blood vessels that immobilize the tumor at this location. Motion trajectories over all respiratory phases exhibit hysteresis effects for both the tumor and the duodenum for all patients (Fig. 5d-e). Duodenum motion was found to be larger in amplitude in all directions compared to tumor motion. Visual inspection of the registered images revealed small residual errors, indicating good motion estimations obtained by OF analysis. Adding all separate difference motion vectors resulted in a mean residual error of $0.49 \mathrm{~mm}$, which is a quantitative measure of the systematic errors. Moreover, the motion trajectories calculated using Elastix (computation time 2:15 hours) revealed similar motion paths, with an average difference below $0.50 \mathrm{~mm}$. Total processing time, which consisted of acquisition (8:35), rebinning/reconstruction (2:44), and 4D motion characterization using optical flow (1:39), was 12:58 minutes.

\section{Discussion}

In this paper, a novel 4D-MRI method was presented using only clinically available scan protocols. The total imaging and processing time was less than 15 minutes, which makes MRI based motion characterization of both the tumor and OARs clinically feasible.

Various 4D motion analyses were performed in three patients. Inter-organ and inter-patient variation was observed in the motion trajectories, which shows the clinical importance of patient specific motion models. Motion heterogeneity was apparent within the tumors, which may be caused by fixation of the tumor to blood vessels, due to ingrowth, which was confirmed by a radiologist.

The calculated patient specific 4D motion trajectories can be used for optimizing the radiation plan for both the GTV and the OARs in abdominal tumors. This can be used to transition from population based internal target volume (ITV) [22] to patient specific ITV. Moreover, the 
ability to perform motion characterization of OARs, such as the duodenum, allows for better definition of planning organs at risk volumes (PRVs) [23], which will aid reducing toxicity. Further, with the development of MR-Linac systems (e.g. [24]), the techniques from this study can be used to calculate the daily variation in motion, online, just prior to treatment and used for online tracking models and beam steering. Although the technique is only used for pancreatic tumors in this study, we believe it can be used for other abdominal tumors, which suffer from respiratory motion, e.g. liver and kidney tumors.

We have shown a 1D MRI navigator correlated better with pancreatic motion, than external respiratory bellows. Moreover, synchronization between the acquired image data and the navigator signal is more robust than for the bellows as mentioned previously [8]. It is worth pointing out, however, that the acquisition of the MR navigator does require some additional scanning time $( \pm 15 \%$ increase in scanning time).

The proposed relative amplitude binning showed the best trade-off between $k$-space filling efficiency and small intra-phase amplitude variation. Phase binning also performed well in the simulations, which is probably caused by the relatively stable respiratory signals used for these simulations. However, when breathing becomes more erratic, phase binning will likely result in larger intra-phase amplitude variation.

Finally, it was shown that radial in-plane sampling is superior to cartesian sampling. In radial sampling the center of $k$-space is sampled for every read-out line, which makes the sequence more robust against image artifacts resulting from undersampling or intra-view motion $[25,26]$. In the reordered data sets this occurs when the bins are not completely filled or when the intra-phase variation is large (Fig. 4).

\subsection{Limitations}


Higher frequency induced motion (e.g. pulsatile motion) will not be visualized by retrospective respiratory binning, which is a limitation of any retrospective method. Furthermore, retrospective binning represents the motion of an average breathing cycle, whereas inter- and intra-cycle variation is not modeled, which may vary substantially (as was seen in the 2D data). Moreover, the overall accuracy of the presented method is very difficult to determine. The retrospective binning, intra-phase amplitude variation and averaging will modulate $k$-space in a complex way. Future studies will therefore mainly focus on investigating the overall accuracy of the proposed technique.

\section{Conclusion}

A way to estimate $3 \mathrm{D}$ motion for ten respiratory phases based on retrospectively binning of $k$-space data using solely clinically scanning protocols has been presented. Optimized respiratory binning, based on an MR navigator, in combination with robust MR sampling resulted in ten $3 \mathrm{D}$ volumes. Using a fast 3D optical flow algorithm, we were able to calculate 4D motion for all abdominal structures within 13 minutes after starting acquisition. 


\section{References}

[1] Keall PJ, Mageras GS, Balter JM, et al. The management of respiratory motion in radiation oncology report of AAPM Task Group 76a. Med Phys 2006;33:3874-3900.

[2] Abdelnour A, Nehmeh S, Pan T, et al. Phase and amplitude binning for 4D-CT imaging. Phys Med Biol 2007;52:3515-3529.

[3] Yamamoto $\mathrm{T}$, Langner U, Loo $\mathrm{Jr} \mathrm{BW}$, et al. Retrospective analysis of artifacts in four-dimensional CT images of 50 abdominal and thoracic radiotherapy patients. Int $J$ Radiat Oncol Biol Phys 2008;72:1250-1258.

[4] Feng M, Balter JM, Normolle D, et al. Characterization of pancreatic tumor motion using cine MRI: surrogates for tumor position should be used with caution. Int J Radiat Oncol Biol Phys 2009;74:884-891.

[5] Bussels B, Goethals L, Feron M, et al. Respiration-induced movement of the upper abdominal organs: a pitfall for the three-dimensional conformal radiation treatment of pancreatic cancer. Radiother Oncol 2003;68:69-74.

[6] Bjerre T, Crijns S, af Rosenschöld PM, et al. Three-dimensional MRI-linac intra-fraction guidance using multiple orthogonal cine-MRI planes. Phys Med Biol 2013;58:4943-4950.

[7] Heerkens HD, van Vulpen M, van den Berg CA, et al. MRI-based tumor motion characterization and gating schemes for radiation therapy of pancreatic cancer. Radiother Oncol 2014; In press.

[8] Tryggestad E, Flammang A, Han-Oh S, et al. Respiration-based sorting of dynamic MRI to derive representative 4D-MRI for radiotherapy planning. Med Phys 2013;40:051909.

[9] Cai J, Chang Z, Wang Z, et al. Four-dimensional magnetic resonance imaging (4D-MRI) using image-based respiratory surrogate: A feasibility study. Med Phys 2011;38:6384-6394.

[10] Ehman RL, Felmlee J. Adaptive technique for high-definition MR imaging of moving structures. Radiology 1989;173:255-263.

[11] Yang J, Cai J, Wang H, et al. Four-Dimensional Magnetic Resonance Imaging Using Axial Body Area as Respiratory Surrogate: Initial Patient Results. Int J Radiat Oncol Biol Phys 2014;88:907-912. 
[12] Von Siebenthal M, Szekely G, Gamper U, Boesiger, et al. 4D MR imaging of respiratory organ motion and its variability. PhysMed Biol 2007;52:1547-1564.

[13] Buerger C, Clough R, King AP, et al. Nonrigid motion modeling of the liver from 3-D undersampled self-gated golden-radial phase encoded MRI. IEEE Trans Med Imaging 2012;31:805-815.

[14] Østergaard Noe K, De Senneville BD, Elstrøm UV, et al. Acceleration and validation of optical flow based deformable registration for image-guided radiotherapy. Acta Oncol 2008;47:1286-1293.

[15] de Senneville BD, Ries M, Maclair G, et al. MR-guided thermotherapy of abdominal organs using a robust PCA-based motion descriptor. IEEE Trans Med Imaging 2011;30:1987-1995.

[16] Roujol S, Ries M, Moonen C, et al. Automatic nonrigid calibration of image registration for real time MR-guided HIFU ablations of mobile organs. IEEE Trans Med Imaging 2011;30:1737-1745.

[17] Stemkens B, Tijssen R, van den Berg C, et al. Optical flow analysis on undersampled radial acquisitions for real-time tracking of the pancreas in MR guided radiotherapy. In Proc Int Soc Magn Reson Med, volume 21 2013; 4325.

[18] Ozhasoglu C, Murphy MJ. Issues in respiratory motion compensation during external-beam radiotherapy. Int J Radiat Oncol Biol Phys 2002;52:1389-1399.

[19] Lu W, Parikh PJ, Hubenschmidt JP, et al. A comparison between amplitude sorting and phase-angle sorting using external respiratory measurement for 4D CT. Med Phys 2006;33:2964-2974.

[20] Wang Z, Bovik AC, Sheikh HR, et al. Image quality assessment: from error visibility to structural similarity. IEEE Trans Image Process 2004;13:600-612.

[21] Klein S, Staring M, Murphy K, et al. Elastix: a toolbox for intensity-based medical image registration. IEEE Trans Med Imaging 2010;29:196-205.

[22] Underberg RW, Lagerwaard FJ, Cuijpers JP, et al. Four-dimensional CT scans for treatment planning in stereotactic radiotherapy for stage I lung cancer. IntJ Radiat Oncol Biol Phys 2004;60:1283-1290. 
[23] McKenzie A, van Herk M, Mijnheer B. Margins for geometric uncertainty around organs at risk in radiotherapy. Radiother Oncol 2002;62:299-307.

[24] Lagendijk JJ, Raaymakers BW, Raaijmakers AJ, et al. MRI/linac integration. Radiother Oncol 2008;86:25-29.

[25] Glover G, Pauly J. Projection reconstruction techniques for reduction of motion effects in MRI. Magn Reson Med 1992;28:275-289.

[26] Scheffler K, Hennig J. Reduced circular field-of-view imaging. Magn Reson Med 1998;40:474-480. 
Figure Legends

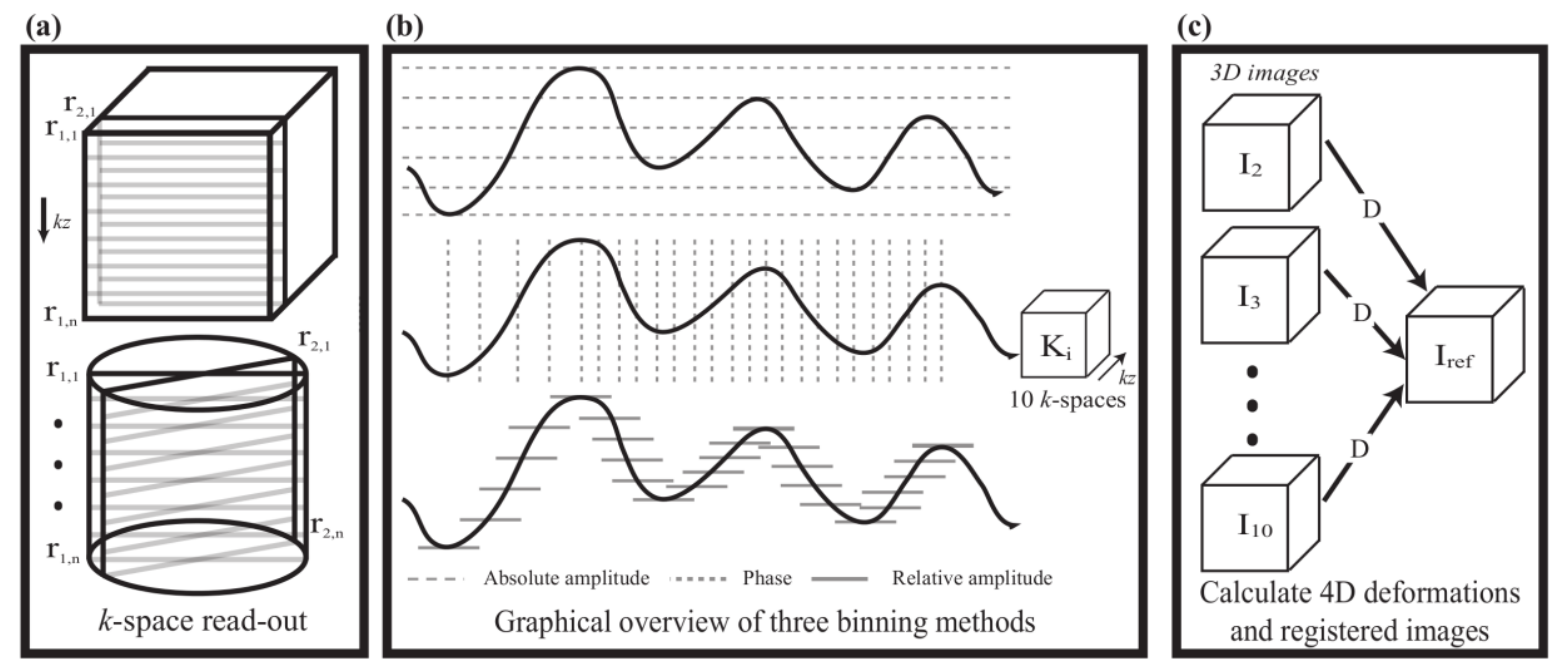

Figure 1 Workflow of the in-vivo experiments. (a) Cartesian and radial trajectories with turbo direction along $k z$. First, read-out lines $\mathrm{r}_{1,1} \ldots \mathrm{r}_{1, \mathrm{n}}$ are acquired within one TFE shot. In the next shot, $r_{2,1} \ldots r_{2, n}$ are acquired, etc. (b) Graphical overview of three binning methods for a respiratory signal. (c) After reconstruction, 3D images are registered to the reference volume, which results in a 4D deformation field. 
(a)

Anatomical image
Residual error maps of GLMs Respiratory bellows Diaphragm navigator

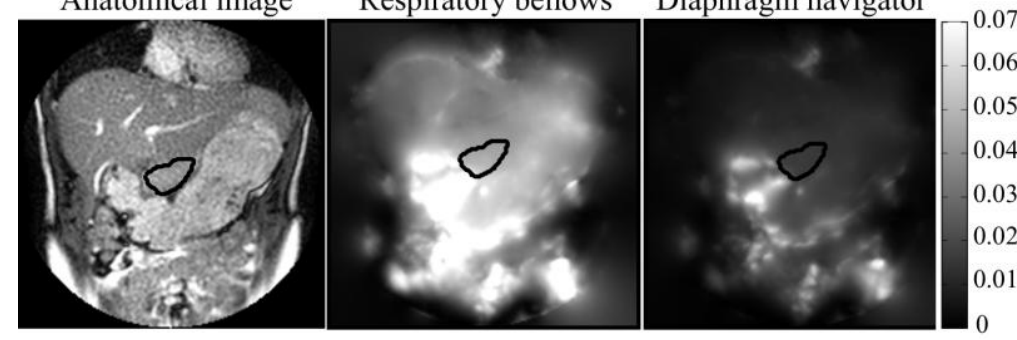

(b)

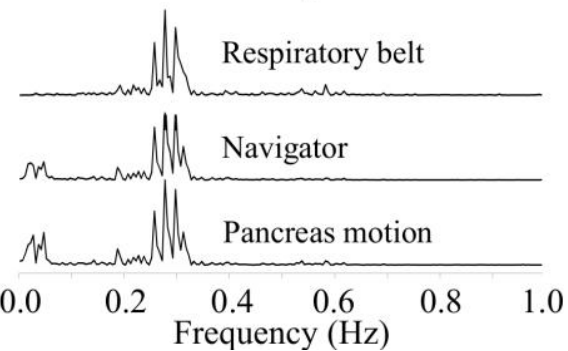

Figure 2 (a) Residual errors of the GLM after regression with both surrogates in one representative subject with the pancreas delineated. (b) Power spectra of the pancreatic motion, navigator and bellows signal (for one subject), showing respiratory peaks between 0.25 and $0.32 \mathrm{~Hz}$ for all modalities, but only low frequency peaks for the pancreatic motion and navigator spectrum. 

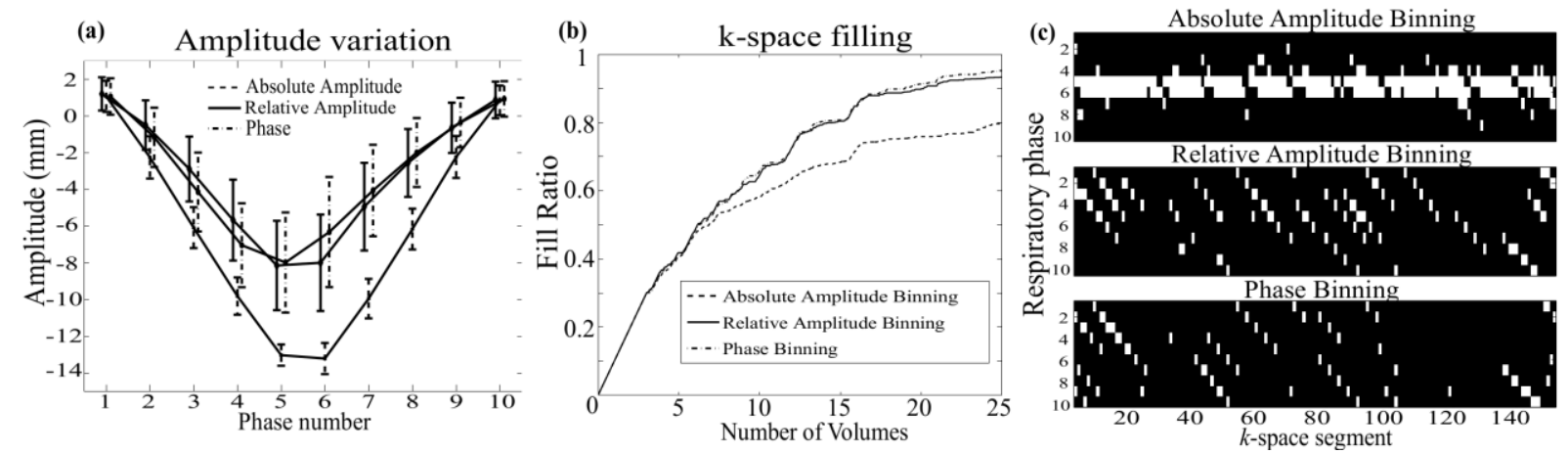

Figure 3 (a) Amplitude variation within the respiratory phases for absolute amplitude, relative amplitude, and phase binning. The error bars display \pm one standard deviation. (b) $\mathrm{k}$-space filling for the three binning methods for an increasing amount of dynamics and (c) corresponding filling of different respiratory phases for absolute amplitude, relative amplitude and phase binning after 25 dynamics. White denotes missing data, black indicates acquired data. 

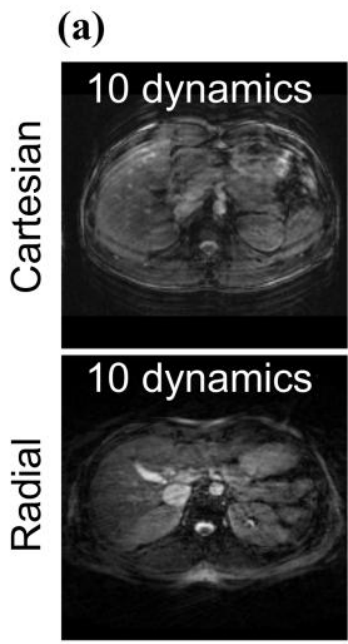

Phase 1

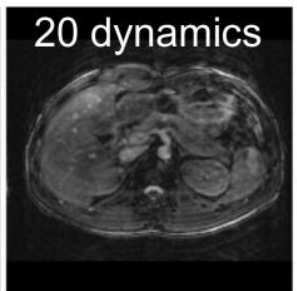

20 dynamics

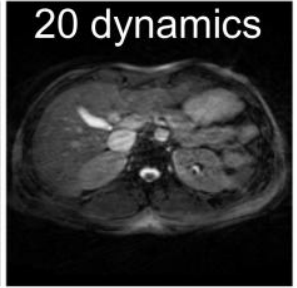

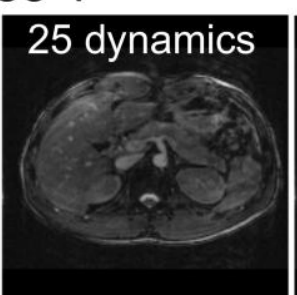

25 dynamics

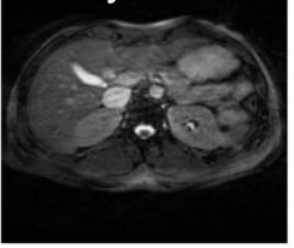

(b) Phase 5
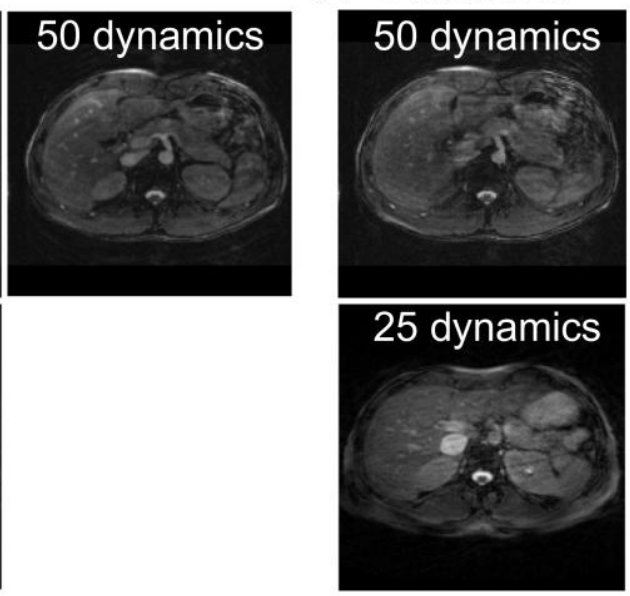

Figure 4 Resulting images for cartesian and radial in-plane sampling. (a) Reconstructions of phase 1 using an increasing number of dynamics, and (b) fully sampled reconstructions of phase 5 . 


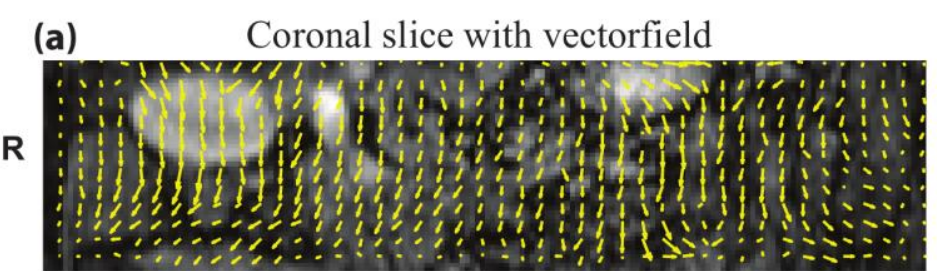

(b)

A

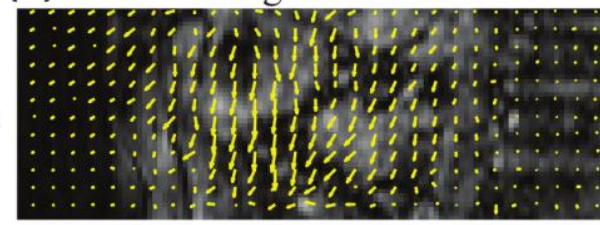

(d)

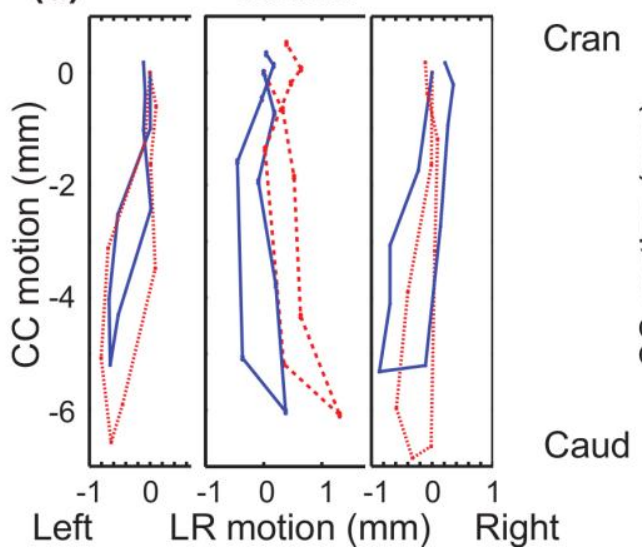

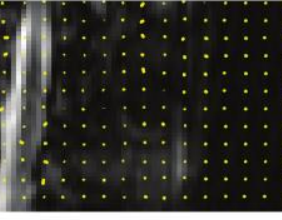

(e)

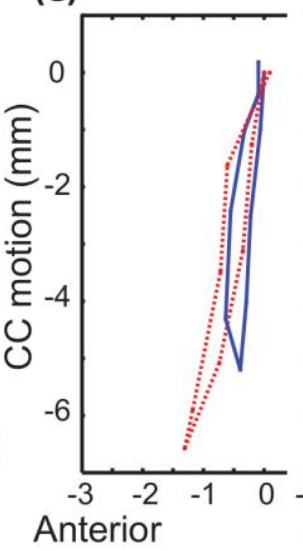

(c) CC-motion on axial slice

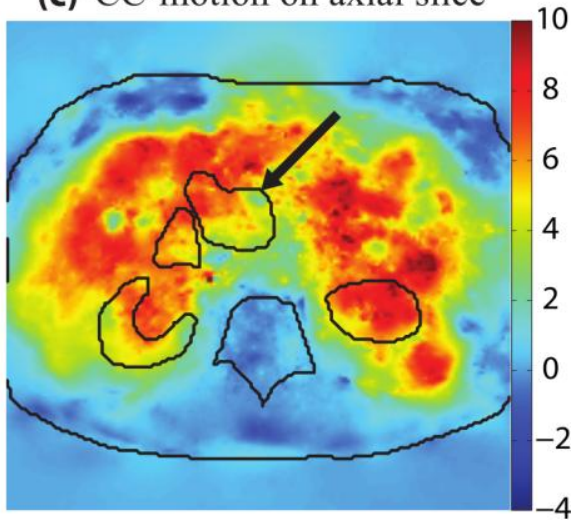

Sagittal

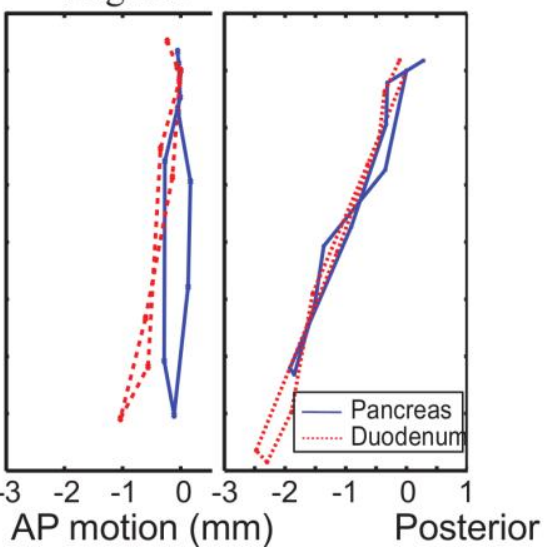

Figure 5 Vectorfields displayed on top of (a) coronal and (b) sagittal slice of the first patient alongside corresponding (c) CC motion with the tumor (arrow) and other structures delineated. (d) Coronal and (e) sagittal motion of tumor and duodenum for three patients. 